\title{
Self-consistent theory of localization and Coulomb drag effect
}

\author{
B. Tanatar \\ Department of Physics, Bilkent University, Bilkent, 06533 Ankara, Turkey \\ A. K. Das \\ Department of Physics, Dalhousie University, Halifax, Nova Scotia, B3H 3J5, Canada
}

(Received 3 January 2000)

\begin{abstract}
We study the Coulomb drag rate for electrons in a double-quantum-well structure in the presence of disorder. The self-consistent theory of localization is used to obtain the frequency dependence of the generalized diffusion coefficient which influences the response functions. The interplay between screening effects and disorder at low temperature gives rise to an enhanced drag rate as the system goes from a weakly localized to a strongly localized phase with increasing disorder. The change in the interlayer momentum transfer rate may be used as a probe to investigate localization properties of coupled quantum-well systems.
\end{abstract}

\section{INTRODUCTION}

There has been extensive theoretical and experimental activity centered around the frictional drag in coupled quantum-well systems in recent years. ${ }^{1}$ The so-called drag effect originates ${ }^{2,3}$ in the interlayer Coulomb interactions between two spatially separated electron systems. When a current $I$ is allowed to pass in only one of the layers, the charge carriers in the second layer are dragged due to the momentum transfer process. Here the distance between the layers is large enough so that tunneling effects are not significant. A drag voltage $V_{D}$ is measured under the condition that no current flows in this second layer. Thus, the transresistance $\rho=V_{D} / I$, or the drag rate $\tau_{\text {drag }}^{-1}=n e^{2} \rho / m^{*}$, probe the Coulomb interaction effects in double-layer electron systems in a transport experiment. The drag effect has been studied experimentally in a variety of setups in which the charge carriers in the quantum wells are electrons, holes, or one of each. $^{4-9}$ The theoretical efforts have concentrated on calculating the momentum transfer rate due to different mechanisms within many-body theory. ${ }^{10-15}$

In this paper, we study the effects of disorder on the Coulomb drag rate in coupled quantum wells in the low temperature regime. In particular, we investigate the changes occurring in the drag rate as the system evolves from a weakly disordered metallic state to a strongly disordered insulating state (Mott insulator). There are several motivations for investigating the disorder effects. The issue of localization in two-dimensional (2D) disordered electron systems is receiving a renewed interest both experimentally and theoretically ${ }^{16,17}$ due to the intricate interplay between disorder and interaction effects. In our work we consider only disorder-induced localization and the metal-insulator transition, but not interaction-induced localization effects. Our disorder is due to nonmagnetic impurities. The experimental results seem to be in agreement with the picture that a metallic phase for weak disorder and an insulating phase for strong disorder exist for an interacting two-dimensional electron gas. ${ }^{18}$ The effects of disorder on the Coulomb drag rate for a double-layer system were first considered by Zheng and MacDonald, ${ }^{10}$ who included disorder in the single-layer density-density response function in the diffusive domain with a constant diffusion coefficient. For a coupled quantumwire system we have recently shown ${ }^{19}$ that the drag rate has a nonmonotonic dependence on the amount of disorder. These works are concerned with the metallic phase for weak disorder. Shimshoni ${ }^{20}$ has suggested that the Coulomb drag rate in double-layer systems offers a possible probe to study the insulating phase for strong disorder. The drag rate is expected to be enhanced at the onset of a metal-insulator transition due to the role played by density fluctuations in both the processes. In the work on coupled quantum-wire systems we used the density-density response function in a number-conserving approximation in order to include weak disorder. This approximation is valid for ranges of $q$ and $\omega$ at the level of the random-phase approximation (RPA), but it is not clear if it can account for strong disorder. The latter is the focus of the work presented here. To this end we have used the density-density response function calculated by Vollhardt and Wölfle ${ }^{21,22}$ in a self-consistent scheme that is capable of treating strong disorder. The expression for the single-layer polarizability $\chi(q, \omega)$ that we use is obtained in a generalized diffusion-pole approximation which is limited to small $q$ and $\omega$ (i.e., $q \ll k_{F}$ and $\omega \ll E_{F}$ ). However, the diffusion coefficient $D(\omega)$ in this calculation is more general than the constant diffusion coefficient used earlier by Zheng and MacDonald $^{10}$ and by us in our work on quantum-wire systems. ${ }^{19}$ We determine $D(\omega)$ numerically in a selfconsistent manner according to the scheme discussed by Vollhardt and Wölfle. ${ }^{22}$ We employ this self-consistent theory of localization ${ }^{22-24}$ to obtain a frequency-dependent diffusion coefficient $D(\omega)$ in the same spirit. The selfconsistent theory of localization has proved useful in predicting the same results as the scaling theory of localization when the interaction effects are ignored. ${ }^{24}$

The rest of the paper is organized as follows. We outline the calculation of the total drag rate in terms of contributions from the ballistic and diffusive regimes in Sec. II. The frequency-dependent diffusion coefficient within the selfconsistent theory of localization is also introduced. In Sec. III we present our results and discuss the possibility of using drag experiments to probe the transition to an insulating 
phase in double-layer systems. We also present an estimate of some physical quantities and compare them with existing experimental results.

\section{THEORY}

We consider two parallel quantum wells of negligible thickness separated by a distance $d$, which is assumed to be large enough so that interlayer tunneling can be neglected. The bare Coulomb interaction between the electrons is given by $V_{i j}(q)=\left(2 \pi e^{2} / \epsilon_{0}\right) e^{-q d\left(1-\delta_{i j}\right)}$, in which $i$ and $j$ label the layers, and $\epsilon_{0}$ is the background dielectric constant. The twodimensional electron density $n$ is related to the Fermi wave vector by $n=k_{F}^{2} / 2 \pi$. We shall use the dimensionless electron gas parameter $r_{s}=\sqrt{2} /\left(k_{F} a_{B}^{*}\right)$, in which $a_{B}^{*}=\epsilon_{0} /\left(e^{2} m^{*}\right)$ is the effective Bohr radius in the semiconducting layer with background dielectric constant $\epsilon_{0}$ and electron effective mass $m^{*}$.

The Coulomb drag rate for double-layer systems has been derived through a variety of theoretical approaches ${ }^{10-15}$ (see also Ref. 1 for a recent review). We adopt the expression given by Zheng and MacDonald. ${ }^{10}$ For simplicity and without loss of generality, we consider the same electron density in both the layers. The drag rate is given by

$$
\tau_{\text {drag }}^{-1}=\frac{1}{2 \pi^{2} m^{*} n T} \int_{0}^{\infty} d q q^{3} \int_{0}^{\infty} d \omega\left|\frac{W_{12}(q, \omega) \operatorname{Im} \chi(q, \omega)}{\sinh (\omega / 2 T)}\right|^{2},
$$

where we have assumed $\hbar$ and $k_{B}=1 . \tau_{\text {drag }}^{-1}$ measures the rate of momentum transferred from one quantum well to the other, and includes the drag rate in the ballistic and diffusive regimes (see below). Here, $\chi(q, \omega)$ is the $2 \mathrm{D}$ dynamic susceptibility, describing the density-density response function of a single-layer electron system. $W_{12}(q, \omega)$ is the dynamically screened effective interaction between electrons in quantum wells 1 and 2 . Within the random-phase approximation, it is given by $W_{12}(q, \omega)=V_{12}(q) / \varepsilon(q, \omega)$, where $\varepsilon(q, \omega)=\left[1-V_{11}(q) \chi(q, \omega)\right]^{2}-\left[V_{12}(q) \chi(q, \omega)\right]^{2}$ is the screening function for the coupled quantum-well system, which uses the bare intra- and interlayer electron-electron interactions (ignoring the correlation effects). Recent numerical calculations ${ }^{15}$ and experimental results ${ }^{9}$ point to the importance of correlations in coupled quantum-well systems, especially in the high temperature regime, viz., $T / E_{F} \sim 0.5$.

In this work, we retain the wave vector, frequency, disorder, and temperature dependence of the dynamic susceptibility $\chi(q, \omega)$ that enters the drag rate expression [Eq. (1)] as well as the screening function $\varepsilon(q, \omega)$. We account for disorder by considering an impurity scattering induced damping $\gamma(=1 / \tau)$ which may be regarded as a phenomenological parameter. Here, $\tau$ is an intralayer (transport) scattering time for electrons, which may be related to the mobility through $\mu=e \tau / m^{*}$. The $2 \mathrm{D}$ dynamical susceptibility is taken to be the noninteracting disorder-free response function in the ballistic regime in which the disorder effects are not thought to be important, i.e., for $q l>1$ and $\omega / \gamma>1$, where $l$ $=k_{F} / \gamma m^{*}$ is the mean free path. In the diffusive regime, the response function is characterized by the generalized diffusion coefficient ${ }^{21,22}$

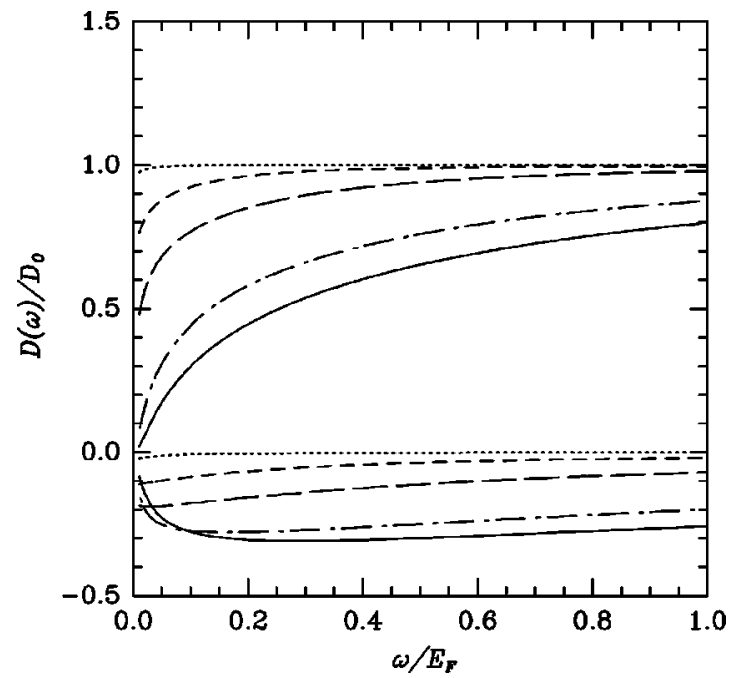

FIG. 1. The real (upper curves) and imaginary (lower curves) parts of the generalized diffusion coefficient $D(\omega)$. The dotted, short dashed, long dashed, dot-dashed, and solid lines indicate $\gamma / E_{F}=0.1,0.5,1.0,2.0$, and 2.5 , respectively.

$$
\chi^{D}(q, \omega)=N_{F} \frac{D(q, \omega) q^{2}}{D(q, \omega) q^{2}-i \omega},
$$

where $D(q, \omega)$ reduces to the commonly used diffusion coefficient $D_{0}=l^{2} \gamma / 2$ in the limit $q l<1$ and $\omega / \gamma<1$. In our work we go beyond a previously used ${ }^{10}$ constant diffusion coefficient, and consider a $D(\omega)$ that retains the full frequency dependence within the self-consistent theory of localization, ${ }^{22}$ the latter being based on a diagrammatic analysis. $D(\omega)$ is obtained from the solution of a selfconsistent equation ${ }^{21,22}$

$$
\frac{D_{0}}{D(\omega)}=1+\frac{1}{\pi N_{F}} \sum_{q} \frac{1}{D(\omega) q^{2}-i \omega}
$$

Although $D(\omega)$ is not as general as $D(q, \omega)$, it contains more physics in the diffusive regime than $D_{0}$. We have solved the above equation numerically for various values of the disorder parameter $\gamma$. The frequency dependence of the real and imaginary parts of $D(\omega)$ is depicted in Fig. 1. It is seen that the frequency behavior of $D(\omega)$ depends crucially on the disorder parameter $\gamma$. For small $\gamma$, corresponding to the metallic phase in the diffusive regime, the real part of $D(\omega)$ is nearly constant, and the imaginary part is vanishingly small. As $\gamma$ increases the frequency dependence of $D(\omega)$ becomes appropriate for a system in the insulating phase. $^{22}$

In view of our objective to study effects of a varying degree of disorder on the drag rate, we find it useful to write the total drag rate as a sum of contributions from the ballistic and diffusive regions, $\tau_{\text {tot }}^{-1}=\tau_{B}^{-1}+\tau_{\Delta}^{-1}$, where

$\tau_{B}^{-1}=\frac{1}{2 \pi^{2} m^{*} n T} \int_{0}^{\infty} d q q^{3} \int_{0}^{\infty} d \omega\left|\frac{W_{12}(q, \omega) \operatorname{Im}\left[\chi^{B}(q, \omega)\right]}{\sinh (\omega / 2 T)}\right|^{2}$,

and 


$$
\begin{aligned}
\tau_{\Delta}^{-1}= & \tau_{D}^{-1}-\frac{1}{2 \pi^{2} m^{*} n T} \int_{0}^{1 / l} d q q^{3} \\
& \times \int_{0}^{\gamma} d \omega\left|\frac{W_{12}(q, \omega) \operatorname{Im}\left[\chi^{B}(q, \omega)\right]}{\sinh (\omega / 2 T)}\right|^{2},
\end{aligned}
$$

with

$$
\begin{aligned}
\tau_{D}^{-1}= & \frac{1}{2 \pi^{2} m^{*} n T} \int_{0}^{1 / l} d q q^{3} \\
& \times \int_{0}^{\gamma} d \omega\left|\frac{W_{12}(q, \omega) \operatorname{Im}\left[\chi^{D}(q, \omega)\right]}{\sinh (\omega / 2 T)}\right|^{2} .
\end{aligned}
$$

The above decomposition, previously given by Zheng and MacDonald, ${ }^{10}$ expresses the total drag rate as the sum of the disorder-free contribution $\tau_{B}^{-1}$ and the correction $\tau_{\Delta}^{-1}$ due to disorder-enhanced fluctuations. In Eqs. (4) and (5), $\chi^{B}(q, \omega)$ is the disorder-free RPA polarizability of a single-layer electron gas. In $\chi^{D}(q, \omega)$, effects of disorder have been included through the frequency-dependent diffusion coefficient $D(\omega)$. For small values of $\gamma$ the system may be considered to be in a weak localization (or metallic) state for which $D(\omega)$ reduces to $D_{0}$. We would like to mention that disorder in the drag effect has also been considered ${ }^{19}$ through a simple number-conserving relaxation-time approximation which gives the response function as

$$
\chi_{\gamma}(q, \omega)=\frac{(\omega+i \gamma) \chi(q, \omega+i \gamma)}{\omega+i \gamma \chi(q, \omega+i \gamma) / \chi(q, 0)}
$$

This reduces to the well-known diffusion pole which is Eq. (2) with $D_{0}$ in the regime $q l<1$ and $\omega / \gamma<1$. Equation (2) with $D(q, \omega)$ approximated as $D(\omega)$ and Eq. (7) have certain complementary features. While Eq. (7) includes the full range of $q$ and $\omega$ within RPA, Eq. (2) contains $D(\omega)$ calculated self-consistently through Eq. (3). With increasing disorder the two-dimensional electron system (on each layer) is expected to enter a strong localization regime. It has been $\operatorname{argued}^{21,22}$ that the latter regime is well described by Eqs. (2) and (3). Our objective is to calculate the drag rate within this self-consistent scheme. We also mention that Eq. (7), as applied to a double-layer system, may not necessarily accommodate a metal-insulator transition as effectively as the selfconsistent calculation is supposed to. The relaxation-time approximation is expected to break down for large $\gamma$, where the self-consistent calculation yields a better description. In the insulating phase, the localization properties are characterized by the localization length $\xi$, defined as ${ }^{22} 1 / \xi$ $=\lim _{\omega \rightarrow 0}[-i \omega / D(\omega)]^{1 / 2}$. We have numerically calculated $\xi$ from the self-consistent solution of $D(\omega)$ [cf. Eq. (3)]. A logarithmic plot of the localization length $\xi$ as a function of the inverse of the disorder parameter $\gamma$ is shown in Fig. 2. We find the expected ${ }^{21,22}$ behaviors of exponentially weak localization for weak disorder (i.e., $\gamma \ll 1$ ), and the powerlaw decay of $\xi$ for strong localization. The results of Fig. 2 suggest that weak to strong localization crossover occurs around $\gamma \simeq E_{F}$.

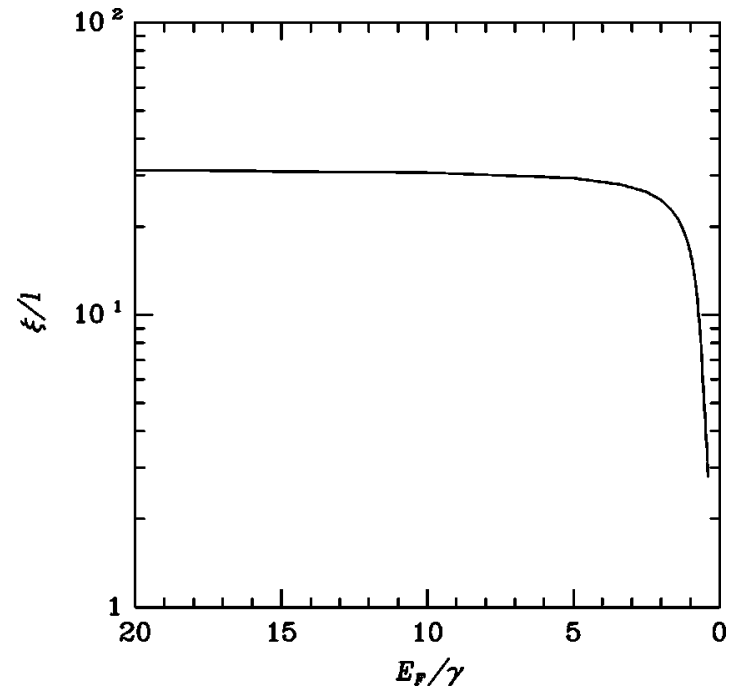

FIG. 2. The localization length as a function of the disorder parameter $\gamma$. The crossover from weak to strong localization is estimated to be $\gamma \approx E_{F}$, when the exponential behavior of $\xi$ goes over to a power-law behavior.

\section{RESULTS AND DISCUSSION}

We use parameters appropriate for a GaAs system for which the recent experiments ${ }^{5-9}$ on the drag rate between coupled quantum-wells were performed, and examine $\tau_{\text {tot }}^{-1}$ at low temperatures. We evaluate $\tau_{\text {tot }}^{-1}$ numerically using the formulation set out in the previous section. The densitydensity response functions that determine the various contributions to the total drag rate enter also in the effective interaction $W_{12}(q, \omega)$ between the electrons in different layers. Our results for $\tau_{\text {tot }}^{-1}$ as a function of temperature are displayed in Fig. 3. We compare our calculations with those of Zheng and MacDonald, ${ }^{10}$ who used the frequencyindependent $D_{0}$ in their description of the diffusive regime. We observe that for small values of disorder $\gamma / E_{F} \ll 1$, both

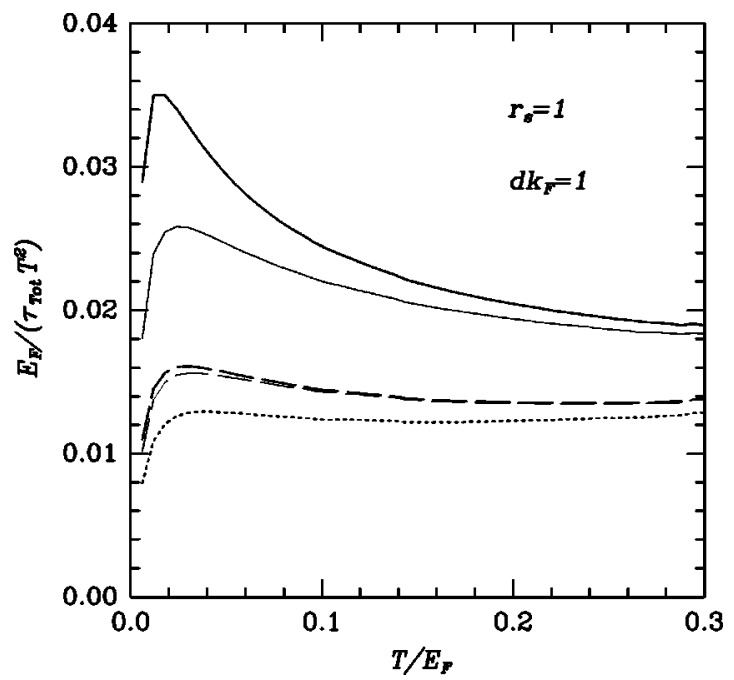

FIG. 3. The scaled total drag rate as a function of temperature. The dotted, dashed, and solid lines indicate $\gamma / E_{F}=0.1,0.5$, and 1.0, respectively. The thick lines are calculated with the frequencydependent diffusion coefficient $D(\omega)$, whereas for the thin lines the frequency-independent $D_{0}$ is used. 


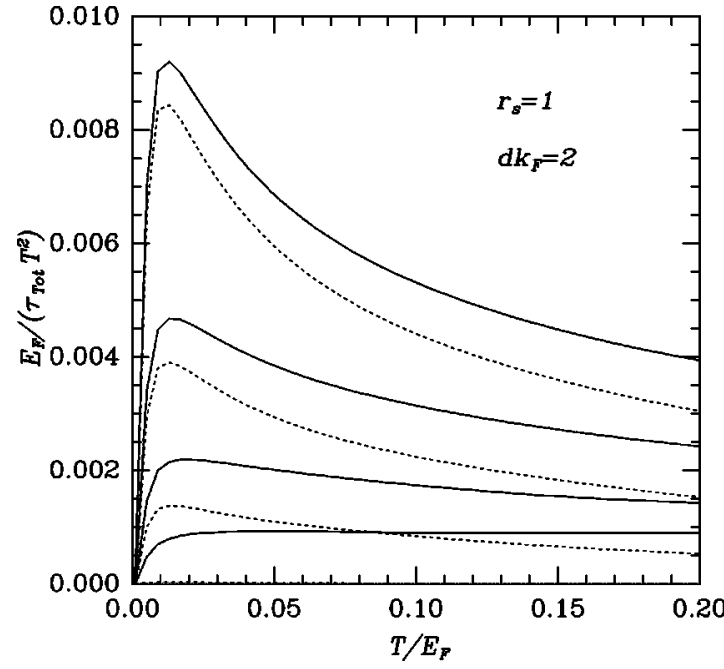

FIG. 4. The scaled drag rate as a function of temperature at $r_{s}$ $=1$ and $d k_{F}=2$. The pairs of curves from bottom to top indicate $\gamma=0.1,0.5,0.75$, and 1 , respectively. The solid and dotted curves are for the total $\tau_{\text {tot }}$ and diffusive $\tau_{\Delta}$ drag rates, respectively.

approaches yield the same result. As $\gamma$ increases the peak structure at low temperatures is greatly enhanced within the frequency-dependent diffusion coefficient treatment. Since for low disorder $\left(\gamma \leqq 0.1 E_{F}\right)$ the dotted curve in Fig. 3 is essentially the same as $\tau_{B}^{-1}$, we find that the self-consistent treatment of disorder effects also indicate a large enhancement over the drag rate in high mobility samples. When the amount of disorder is further increased we observe that the drag rate starts to decrease, showing a nonmonotonic behavior. Such a behavior was also noted for double-wire systems within the relaxation-time approximation. ${ }^{19}$

To discern the amount of contribution to the total drag rate coming from the diffusive regime, we show in Fig. 4 the total drag rate $\tau_{\text {tot }}^{-1}$ and the correction due to enhanced fluctuations at the long wavelength and low frequency region $\tau_{\Delta}^{-1}$. We find that for small $\gamma$ the electrons can be thought of as largely in the ballistic regime. As $\gamma$ gradually increases, the contribution from $\tau_{\Delta}^{-1}$ increases appreciably. The drag experiments $^{5-8}$ were done on GaAs-based materials. Using the material parameters (effective mass $m^{*}=0.07 m_{e}$, dielectric constant $\epsilon_{0}=13$ ) of GaAs, the density parameters $r_{s}=1$ and $r_{s}=2$ assumed in Figs. 3 and 4 correspond to $n$ $\approx 3 \times 10^{11} \mathrm{~cm}^{-2}$ and $n \approx 0.7 \times 10^{11} \mathrm{~cm}^{-2}$, respectively. The quantum-well separation distances $d=k_{F}^{-1} \simeq 140 \AA$ and $d$ $=2 k_{F}^{-1} \simeq 280 \AA$ (for $r_{s}=1$ ) are somewhat lower than the experimental values, which are in the range $d \gtrsim 500 \AA$ (we consider large $d$ cases in the following figures). The mean free path $l=2 k_{F}-1 /\left(\gamma / E_{F}\right)$ decreases from $l \simeq 2000 \AA$ to $l$ $\simeq 200 \AA$ when the disorder parameter increases from $\gamma$ $=0.1 E_{F}$ to $\gamma=E_{F}$. The predicted drag enhancement would occur at $T \simeq 2 \mathrm{~K}\left(T=0.03 E_{F}\right.$, with $\left.E_{F} \approx 70 \mathrm{~K}\right)$ at a peak value $\tau_{\text {tot }}^{-1} \approx 2 \times 10^{8} \mathrm{~s}^{-1}$. This is two orders of magnitude greater than the drag rate observed for high mobility samples. Similar estimates were also given by Shimshoni. ${ }^{20}$ The low temperature drag rate (or resistivity) in the experiments has a peak due to virtual phonons. ${ }^{5,25}$ To make more precise comparison with experiments (using low mobility samples) the phonon contribution should be subtracted out.
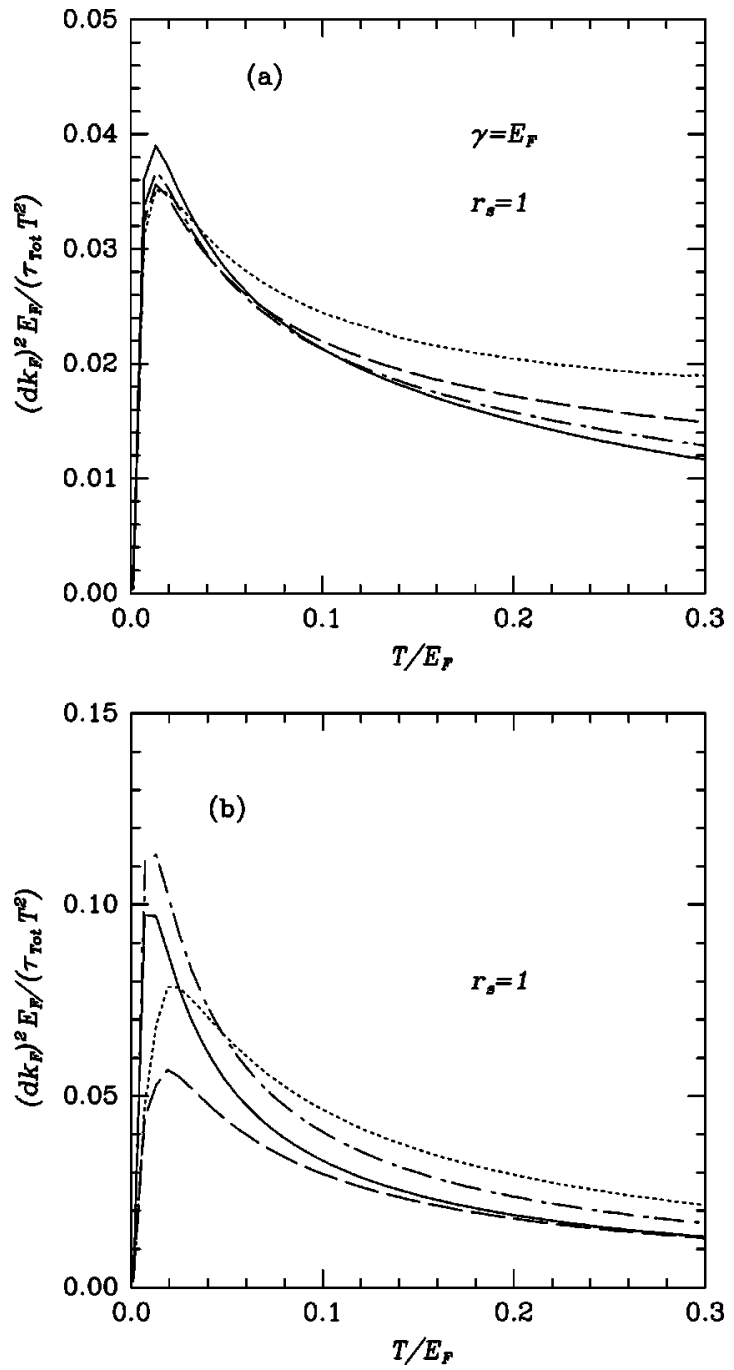

FIG. 5. (a) The scaled total drag rate as a function of temperature. The dotted, dashed, dot-dashed, and solid lines indicate $d k_{F}$ $=1,1.5,2$, and 3 , respectively. The disorder and density parameters are such that $\gamma / E_{F}=1$ and $r_{s}=1$. (b) The total drag rate for the insulating regime. The solid, dot-dashed, dashed, and dotted lines are for $\left(d k_{F}=10, \gamma / E_{F}=2\right), \quad\left(d k_{F}=8, \gamma / E_{F}=2\right), \quad\left(d k_{F}\right.$ $\left.=6, \gamma / E_{F}=3\right)$, and $\left(d k_{F}=4, \gamma / E_{F}=3\right)$, respectively.

We investigate the scaling property of the total drag rate with respect to the interlayer separation distance $d$ in Fig. 5 . Shimshoni ${ }^{20}$ distinguished the cases of $\xi \ll d$ (deep in the insulating state) and $\xi \gg d$ (metallic regime), in the analysis of the drag rate in the Mott insulator state. In the calculations shown in Fig. 5(a), the parameters are such that $\xi \gtrsim d$; thus the system may be considered to be in the vicinity of the metal-insulator transition. Our results largely obey the $d^{-2}$ dependence of the transresistivity formula given by Shimshoni ${ }^{20}$ in this regime. In Fig. 5(b) we show the scaled total drag rate for various combinations of $\gamma$ and $d$ such that $\xi<d$ where the system is well in the insulating state. We note that the drag rate again displays a $d^{-2}$-dependent scaling, different from the $d^{-6}$ dependence predicted by Shimshoni ${ }^{20}$ for the same regime. The origin of this discrepancy could be that for the parameters used a much larger value of $d$ must be chosen, or that the frequency-dependent diffusion coefficient which also enters the dielectric function in $W_{12}(q, \omega)$ alters the $d$ dependence. In the absence of any 


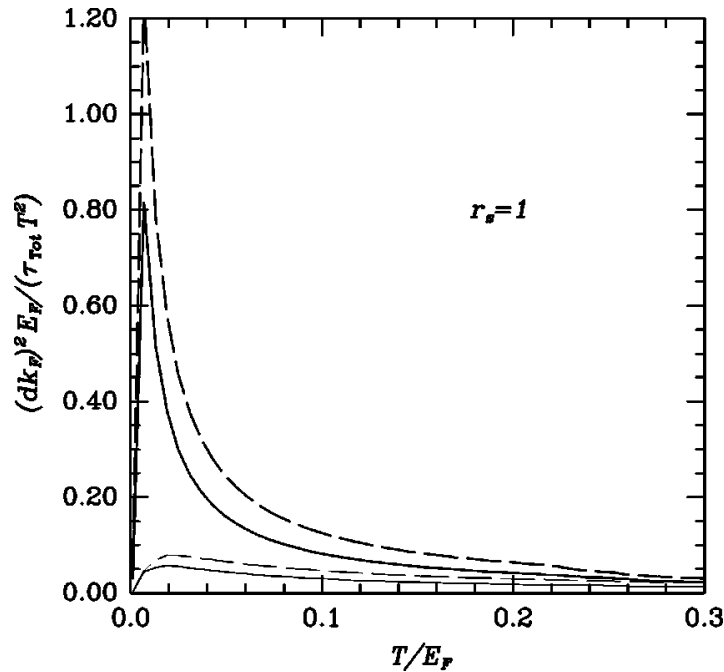

FIG. 6. The total drag rate calculated in the dynamic (thick lines) and static (thin lines) screening approximations. The solid and dashed lines indicate $\left(d k_{F}=4, \gamma / E_{F}=3\right)$ and $\left(d k_{F}=6, \gamma / E_{F}\right.$ $=3$ ), respectively.

systematic experimental data we report our findings as they emerge from the self-consistent calculation of the diffusion coefficient. We examine the influence of dynamical correlations on the drag rate in Fig. 6. The dielectric function $\varepsilon(q, \omega)$ is included in full (thick lines) and in the static approximation, i.e., $\varepsilon(q)$, (thin lines). We observe that the dynamical nature of $D(\omega)$ and hence $\varepsilon(q, \omega)$ contribute significantly to $\tau_{\text {tot }}^{-1}$ at low temperatures.

The low temperature behavior of the drag rate for coupled quantum wells has been considered by many researchers. Zheng and MacDonald ${ }^{10}$ split the contributions of ballistic and diffusive regimes and calculated the correction to the interlayer scattering rate due to disorder-enhanced interactions. Our work is similar in spirit, except we include the frequency dependence of the diffusion coefficient. The same enhancement in the drag resistivity was also predicted by Kamenev and Oreg $^{14}$ using diagrammatic perturbation theory. Gornyi, Yashenkin, and Khveshchenko ${ }^{26}$ studied the effects of correlated disorder in the form of an impurity potential in the barrier region between the quantum wells. Such an approach would be relevant to the actual systems if controlled experiments can be performed. In a recent paper, Shimshoni $\mathrm{i}^{20}$ considered the Coulomb drag between two parallel layers in the Anderson insulating state, examining the Mott and Efros-Shklovskii types separately. In the analysis, Shimshoni ${ }^{20}$ found that $\rho_{D}$ is suppressed for a Mott insulator with decreasing localization length (i.e., increasing disorder). In all these attempts the disorder has the effect of enhancing $\tau_{\text {drag }}^{-1}\left(\right.$ or $\tau_{\text {tot }}^{-1}$ ) or the transresistance $\rho$ as a function of $T$ for low to moderate disorder while suppressing $\tau_{\text {drag }}^{-1}$ for large disorder. In a Boltzmann-theory-based calculation of the drag rate Flensberg and $\mathrm{Hu}^{13}$ found that charged impurities located a distance $s$ away from the quantum wells influenced $\tau_{D}^{-1}$ significantly for $s \lesssim 400 \AA$. Classical simulations to determine the influence of ionized impurities on Coulomb drag have also been performed. ${ }^{27}$ Our calculations indicate similar qualitative results without specifying the nature of disorder. We also point out that disorder effects in Coulomb drag problems are gaining attention recently in a variety of related contexts. $^{28}$

As the electron density is decreased the exchangecorrelation effects become significant and the RPA we adopt will be less satisfactory. In studies of drag resistivity and drag rate in double-layer systems it has been found important to include correlation effects beyond those described by the RPA to improve agreement with experimental data at low densities. ${ }^{15}$ In this work, we have kept the electron gas parameter low $\left(r_{s}=1\right)$ so that the RPA should be adequate. The interplay between the disorder and correlation effects would have to be taken into account in a more consistent and systematic viewpoint for larger $r_{s}$ values. The interaction effects on the Anderson transition in disordered systems have been discussed by Sadovskii. ${ }^{29}$

In summary, we have considered the Coulomb drag effect between two parallel quantum wells in the presence of disorder treated phenomenologically. The temperature dependence of the drag rate is known to be significantly enhanced with increasing disorder as the system evolves from a metallic state toward an insulating state. Using the self-consistent theory of localization to deduce the frequency dependence of the diffusion coefficient, we account for the influence of disorder on the density-density correlation function. We find that for small $\gamma$ the drag rate is further increased. For larger values of $\gamma$, the density fluctuations are suppressed with a reduced localization length and the drag rate is reduced. Thus, the drag rate $\tau_{\text {drag }}^{-1}$ exhibits a nonmonotonic behavior with respect to the strength of disorder, and may be used as a possible probe to understand the localization properties in Coulomb coupled systems. A systematic experimental study with varying degrees of disorder using low mobility samples should be able to test some of our predictions.

\section{ACKNOWLEDGMENTS}

This work is partially supported by the Scientific and Technical Research Council of Turkey (TUBITAK) under Grant No. TBAG-1662 and NATO under Grant No. SfP971970. We thank Professor M. V. Sadovskii and Professor I. O. Kulik for fruitful discussions.
${ }^{1}$ The field was reviewed recently by A.G. Rojo, J. Phys.: Condens. Matter 11, R31 (1999).

${ }^{2}$ M.B. Pogrebinskii, Fiz. Tekh. Poluprovodn. 11, 637 (1977) [ Sov. Phys. Semicond. 11, 372 (1977)].

${ }^{3}$ P.J. Price, Physica B \& C 117, 750 (1983).

${ }^{4}$ P.M. Solomon, P.J. Price, D.J. Frank, and D.C. La Tulipe, Phys.
Rev. Lett. 63, 2508 (1989).

${ }^{5}$ T.J. Gramila, J.P. Eisenstein, A.H. MacDonald, L.N. Pfeiffer, and K.W. West, Phys. Rev. Lett. 66, 1216 (1991); Phys. Rev. B 47, 12957 (1993).

${ }^{6}$ U. Sivan, P.M. Solomon, and H. Shtrikman, Phys. Rev. Lett. 68, 1196 (1992). 
${ }^{7}$ H. Rubel, E.H. Linfield, D.A. Ritchie, K.M. Brown, M. Pepper, and G.A.C. Jones, Semicond. Sci. Technol. 10, 1229 (1995).

${ }^{8}$ N.P.R. Hill, J.T. Nicholls, E.H. Linfield, M. Pepper, D.A. Ritchie, G.A.C. Jones, B.Y.-K. Hu, and K. Flensberg, Phys. Rev. Lett. 78, 2204 (1997).

${ }^{9}$ H. Noh, S. Zelakiewicz, X.G. Feng, T.J. Gramila, L.N. Pfeiffer, and K.W. West, Phys. Rev. B 58, 12621 (1998).

${ }^{10}$ L. Zheng and A.H. MacDonald, Phys. Rev. B 48, 8203 (1993).

${ }^{11}$ A.-P. Jauho and H. Smith, Phys. Rev. B 47, 4420 (1993).

${ }^{12}$ H.C. Tso, P. Vasilopoulos, and F.M. Peeters, Phys. Rev. Lett. 68, 2516 (1992); 70, 2146 (1993).

${ }^{13}$ K. Flensberg and B.Y.-K. Hu, Phys. Rev. Lett. 73, 3572 (1994); Phys. Rev. B 52, 14796 (1995).

${ }^{14}$ A. Kamenev and Y. Oreg, Phys. Rev. B 52, 7516 (1995); K. Flensberg, B.Y.-K. Hu, A.-P. Jauho, and J.M. Kinaret, ibid. 52, 14761 (1995).

${ }^{15}$ L. Świerkowski, J. Szymański, and Z.W. Gortel, Phys. Rev. B 55, 2280 (1997); Phys. Rev. Lett. 74, 3245 (1995).

${ }^{16}$ S.V. Kravchenko, G.V. Kravchenko, J.E. Furneaux, V.M. Pudalov, and M. D'Iorio, Phys. Rev. B 50, 8039 (1994); D. Popović, A.B. Fowler, and S. Washburn, Phys. Rev. Lett. 79, 1543 (1997)

${ }^{17}$ C. Castellani, C. Di Castro, and P.A. Lee, Phys. Rev. B 57, 9381 (1998); Q. Si and C.M. Varma, Phys. Rev. Lett. 81, 4951
(1998); D. Belitz and T.R. Kirkpatrick, Phys. Rev. B 58, 8214 (1998).

${ }^{18}$ A. Gold and W. Götze, Phys. Rev. B 33, 2495 (1986).

${ }^{19}$ B. Tanatar, Phys. Rev. B 58, 1154 (1998).

${ }^{20}$ E. Shimshoni, Phys. Rev. B 56, 13301 (1997).

${ }^{21}$ D. Vollhardt and P. Wölfle, Phys. Rev. B 22, 4666 (1980); Phys. Rev. Lett. 48, 699 (1982).

${ }^{22}$ D. Vollhardt and P. Wölfle, in Electronic Phase Transitions, edited by W. Hanke and Yu.V. Kopaev (North-Holland, Amsterdam, 1990).

${ }^{23}$ W. Götze, Philos. Mag. B 43, 219 (1981).

${ }^{24}$ P.A. Lee and T.V. Ramakrishnan, Rev. Mod. Phys. 57, 287 (1985); D. Belitz and T.R. Kirkpatrick, ibid. 66, 261 (1994).

${ }^{25}$ H. Noh, S. Zelakiewicz, T.J. Gramila, L.N. Pfeiffer, and K.W. West, Phys. Rev. B 59, 13114 (1999); S.M. Badalyan and U. Rossleri, ibid. 59, 5643 (1999); B.Y.-K. Hu, ibid. 57, 12345 (1998).

${ }^{26}$ I.V. Gornyi, A.G. Yashenkin, and D.V. Khveshchenko, Phys. Rev. Lett. 83, 152 (1999).

${ }^{27}$ V. Cambel and M. Moško, Semicond. Sci. Technol. 8, 364 (1993).

${ }^{28}$ Y. Oreg and A. Kamenev, Phys. Rev. Lett. 80, 2421 (1998); A. Stern and I. Ussishkin, Physica E 1, 176 (1997); J. Baker, G. Vignale, and A.G. Rojo, Phys. Rev. B 60, 8804 (1999).

${ }^{29}$ M.V. Sadovskii, Phys. Rep. 282, 225 (1997). 